\title{
A NANODIAMOND FOR STRUCTURAL BIOMIMETIC SCAFFOLDS
}

\author{
Raffaella Aversa $^{1}$ - Relly Victoria V. Petrescu ${ }^{2}$ - Antonio Apicella ${ }^{1}$ - Florian Ion T. Petrescu ${ }^{3 *}$ \\ ${ }^{1}$ Advanced Material Lab, Department of Architecture and Industrial Design, University of Campania, Luigi Vanvitelli \\ Aversa 81031 (CE) Italy \\ ${ }^{2}$ Transport Traffic and Logistics Department, Bucharest Polytechnic University, Bucharest 060042 (CE) Romania \\ ${ }^{3}$ Theory of Mechanisms and Robots Department, Bucharest Polytechnic University, Bucharest 060042 (CE) Romania
}

\begin{tabular}{l} 
ARTICLE INFO \\
\hline Article history: \\
Received: 19.02 .2017$. \\
Received in revised form: 5.6.2017 \\
Accepted: 21.07 .2017$. \\
\hline Keywords: \\
Hybrid nano-composites \\
Biomaterials \\
Bioactive scaffolds \\
Bioengineering \\
Biomedical \\
\hline
\end{tabular}

DOI: http://doi.org/10.30765/er.39.1.9

\section{Introduction}

Traditional orthopaedic prostheses are mainly made of metals and ceramics materials with outstanding strength and rigidity properties but with a high physiological invasiveness. These systems, while guarantying mechanical functional tenure, they often strongly physiologically and mechanically interfere with human bone. This unwanted

\begin{abstract}
:
Bio-mechanically active scaffolds for tissue engineering combining hydrophilic polymeric matrix and nano-diamond fillers properties are presented and discussed in this paper. The resulting scaffolding materials revealed remarkable mechanical and biological properties to be exploited in advanced biomedical applications. The novel hybrid material is based on 2 and 5 volume \% of detonation nano-diamond particles in a hydrophilic poly-(hydroxyl-ethylmethacrylate) matrix. According to its mechanical and biological properties, the nanocomposite shows a hybrid nature. The base analytical procedures for the preparation of the hybrid nanocomposites and some preliminary mechanical characteristics are presented. The proposed hybrid system has been considered for potential biomimetic, osteoconductive and osteoinductive scaffolds application in bio-mechanically active bone scaffolds for osteoblast and stem cell differentiation and growth. These more rigid hybrid nano-composites are predicted to possess improved mechanical strength overcoming the mechanical weaknesses of traditional hydrogels clinically utilized for bone regeneration.
\end{abstract}

behaviour derives from the fact that bones are complex functional and structural entities composed both by less rigid open-cell trabecular and more dense and rigid materials that are combined, at medullar and cortical bone levels, to achieve specific rigidities and strength. Failures and longterm unreliability follow from a not full integration with the bone local physiology and with patient personal characteristics. In particular, the traditional

\footnotetext{
* Corresponding author

E-mail address: fitpetrescu@gmail.com
} 
prosthetic systems, which are not able to completely mimic all the healthy bone functions, become particularly not adequate for younger patients who have higher life expectations and then high prostheses requirements in terms of duration, biomechanical osseointegration, and low invasiveness. The bone-prosthesis interferences have been recognized to occur at two dimensional levels, namely, at micro and macro scale. The first (at a micro-scale) accounts for the biological and micromechanical interactions of the synthetic biomaterial with the bone forming cells and their adaptive properties, while the second (at a macro scale) determines the prosthesis full biomechanical functionality and ability to restore the biological stress state distribution in the prosthesized bone.

New bio-mechanically active materials with mechanical properties able to withstand loads without mechanically interfere with the healthy bone physiology are then needed. The bone orthotropy and complex structure should be accounted and preserved in order to recreate the same stresses and strains distribution. The use of micro-trabecular metallic prostheses and scaffolds simulating the behavior of cancellous and cortical bone has been proposed to recreate the macro distribution of stresses and deformations in the bone. However, a suitable biometric interface is needed at micro level where osteon and osteoblast cell interact. The development of polymeric hybrid nano-composites has been proposed in recent studies. These hybrid materials have shown to posses the necessary mechanical and biological properties to favor local healthy bone generations. Therefore, specific and tunable properties simulating the bone tissues are required at micro level of the interface to guide bone-forming cells to growth and successful integrate with the bonegrafting material in the host tissue. The main reasons for using bone scaffolds are thus to provide an environment for bone formation, maintain space, and provide mechanical support to the bone skeleton during the repair process. Biomaterials science today is a highly interdisciplinary field playing a central role in the development of regenerative tissue engineering applications. Tight cooperation among biologists, chemists, material engineers, physicists, and clinician raced research in this area to a level that is favoring the development of new biomaterials successfully fronting the different request of different tissue-engineering specifications. Even if more investigation have to be implemented, many biomaterials applications are near to clinical application, and regenerative medicine has greatly improved and it will guarantee more applications from bench-to-the-bedside in near future [1-6].

Graphite, which is one of the allotropic forms of Carbon, is thermodynamically more stable at ambient temperatures and pressures, conversely, the diamond allotropic form is more stable at high pressures and temperature and it can exist only in its metastable state at ambient conditions. The differences in stability of allotropic forms are a consequence of the high-energy barrier that separated the graphitic $\mathrm{Sp}_{2}$ (Left in Figure 1) and the diamond $\mathrm{Sp}_{3}$ configuration (Right in Figure 1).
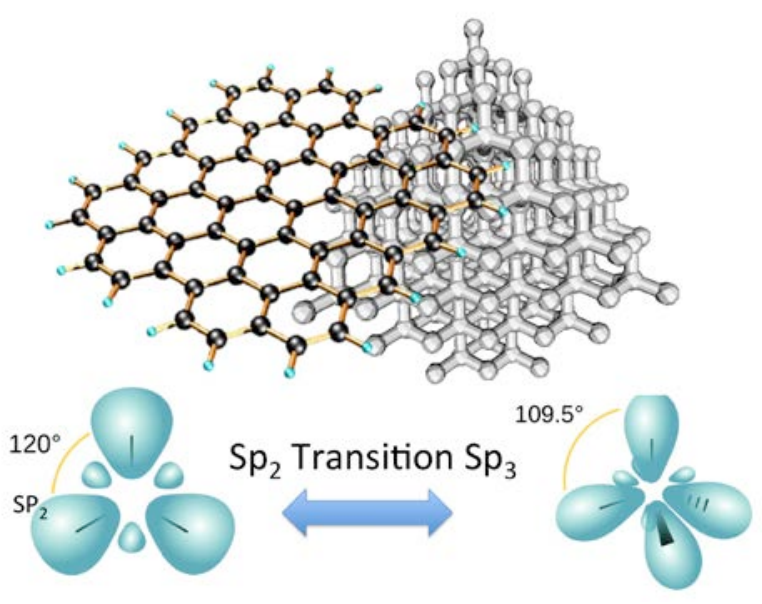

Figure 1.Left) Graphite (SP2 hybridization) and Right) Diamond (SP3 hybridization) Carbon allotropic forms.

Therefore, the transformation of graphite in diamond requires high temperatures and pressures in presence of catalysts. However, at nano-size level, a third equilibrium parameter related to the surface area becomes crucial and relevant in the system equilibrium energy distribution: Gibbs free energy is significantly influenced by the contribution of the surface energy, leading to changes in the thermodynamic equilibrium phase diagram [7-10]. Atomistic models demonstrated that nano-diamonds of $3 \mathrm{~nm}$ with tetrahedral hydrocarbons are more stable than graphite in its poly-aromatics form [7].

It is interesting and it opens a compelling technological application- the fact that a more complex structure is generated at the nano-diamond 
interface. Cuboctahedral clusters presenting a transition from $\mathrm{Sp}_{3}$ to $\mathrm{Sp}_{2}$ carbons at their surfaces in the case of atom cluster of 1.0 - $3.0 \mathrm{~nm}$ have been reported by Barnard and Sternberg [9].

Reversible nanodiamond-graphitic carbon onion like phase transformation has been observed by Xiao et al [11] occuring in this morphological transition interface even at room temperature and pressure leading to the formation of bucky-diamond characterized by diamond cores with graphitic shells (Figure 2) [9].

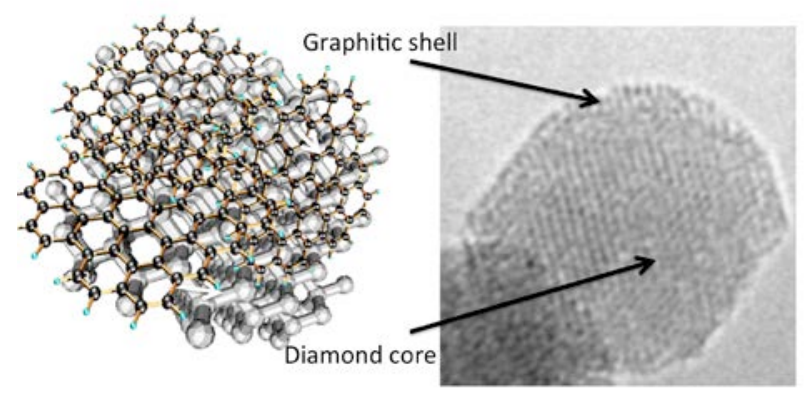

Figure 2. Nano-diamond core with external graphitic shell (left side) and (TEM of a detonation nano-diamond atomic structure (right side).

According to these findings, nano-diamond surfaces can be modified to form functionalised cyclohexene systems using the well assessed chemistry of graphitic carbon, i.e. the Diels-Alder cycloaddition reactions between conjugated diene and dienophile, [12].

New perspectives open to these materials based on nano-crystalline Sp2 and Sp3 Carbon structures for future nano-technological development in biomedical structural applications. Such nanocrystalline particles are produced by detonation of carbon explosive materials [13-16] and, hence, are reported as detonation nano-diamond with characteristic sizes of 3-5 nm. Simple applications in lubricants, galvanic coatings, polymer nanocomposities, polishing systems, have been initially exploited for detonation nano-diamond. However, new and more valuable niche applications have recently arisen in the area of electronics, diamond ceramics production, emission devices, catalysts and fuel cells as proton-conducting nanocomposite membranes. Preliminary clinical and biochemical investigation have shown that these detonation nano-diamonds are biocompatible and non-toxic, opening for new bio-medical applications both considering its easy controllable variety of possible surface chemistry and their intrinsic high mechanical characteristics.

Detonation nano-diamonds, which surface is characterized by different levels of purity and presence of undesired functional groups/elements, may not be directly suitable for biomedical applications where high surface chemical purity and surfaces chemical uniformity are needed [17-18]. After rough production, these materials undergo several purification procedures. A simple method utilizes surface oxidation and different levels of purities and surface properties can be obtained. Oxidation carried out at high temperatures in air/Ozone atmosphere can yield to purification of Carbon fraction not present as diamond up to 95 per cent by weight [19-20].

The oxidation of the nano-diamond surfaces other than removing undesired processing functional compounds forms oxygen-containing groups (red dots in Figure 2), such as anhydrides and carboxylic acids [20], that are suitable for hydrogen or polar secondary bonding with suitable species.

By air/ozone purification, then, we can functionalize the surface obtaining carboxylated nano-diamond with highly reactive and hydrophilic surface $\mathrm{OH}$ terminations, which are highly appropriate for biomedical applications [21-22].

However, the toxicity of nano-diamonds remains controversial and it is a real concern [23]. In vitro and in vivo further investigation are then needed to evaluate characteristics such as in vivo mechanical and physiological behaviors [23-28], cell viability, and undesired gene modification activity. Although biocompatibility and adverse effect were also described in literature for the use of nanosilica particles, our published investigations of have shown that nano-composite and hybrid materials made combining amorphous silica nanoparticles reached high level of biocompatibility and bioactivity that were not reached by pure nano and micro silica, and by the p-HEMA matrix. These hydrogel hybrid nano-composites have been tested for water sorption, swelling in water and in isotonic saline solution, and for the fibroblasts and osteoblasts as cell response with assays of adhesion, distribution, and morphology. The presence of the polymer-linked silica makes this biomaterials excellent, with respect to the pHEMA alone. Good properties of osteoinduction have been also 
observed for differentiation of dental pulp stem cells.

Nanotechnology applied to micro and nanomaterials bioengineering is being gradually accepted in emerging applications where coatings or three-dimensional scaffolds are needed [29-33]. The creation of ideal bone scaffolds is a growing argument of research. Such ideal scaffold has to provide a rigid and resilient network to temporarily substitute the damaged bone function while creating a bioactive substrate for bone regeneration. At the same time it should be able to fully integrate with it [1, 4, 6, 34].

These self assembled nanostructured composite were also micro-foamed and tested as peri-implantar scaffold to host osteoblast grow factors or stem cells for osteoblasts differentiation [35].

These new hybrid materials have been show to be able to biomechanically stimulate bone growth in the range of physiological strains that permit the healthy growth permitting an early and organized full integration of the implant in the receiving bone. This effect was primarily due to the micromechanical stress and strain states that generate at level of the bone/implant interface.

\section{Materials and methods}

Raw detonation nanodiamonds (Aldrich, $\geq 97 \%$ ) with a mean diameter of 3-5 $\mathrm{nm}$ and specific surface area of $400 \mathrm{~m}^{2} \cdot \mathrm{g}^{-1}$ were utilized as bioactive filler. First detonation nanodiamond hydrophilization has been carried out according to Shenderova technique [29]. Surface graphitization with subsequent functionalization allows dispersion into $20-\mathrm{nm}$ aggregates. High-temperature hydrogen treatment resulted in stable single-particle aqueous nanodiamond colloids from which a fraction of 2-4$\mathrm{nm}$ nanodiamonds was isolated by centrifugation at $>10,000$ rpm [20].

The resulting nanodispersion was then mixed with 2-hydroxyethyl methacrylate (HEMA) (SigmaAldrich Chemicals Co., St. Louis, MO, USA). HEMA monomers-nanodiamond suspension (Figure 2) has been then radical polymerized in presence of the $\boldsymbol{\alpha}-\boldsymbol{\alpha}^{\prime}$ azoisobutyrronitrile (AIBN, Fluka Milan, Italy) thermal initiator.

Nanocarbon contents at 2 and 5\% by volume have been prepared.

The degassed reactive suspension was first transferred into $2.0 \mathrm{~mm}$ thick planar moulds and then polymerized at $60^{\circ} \mathrm{C}$ for $24 \mathrm{~h}$. A post-cure of 1 $\mathrm{h}$ at $90^{\circ} \mathrm{C}$ was finally carried out.

Preliminary Dynamic Mechanical Tests have been run according to ref [29] for samples at 2 and 4\% volume fractions.

\section{Results and discussion}

The presence of functionalised oxidized Detonation nano-diamonds in the monomer reacting mixture favour the self orientation of HEMA polar monomers. The nanoparticles, in fact, are characterized by the presence of oxygen-containing groups that leads to a preferential orientation and self assembly of the HEMA monomer hydroxyl groups toward the nano-filler surface (Fig. 3)

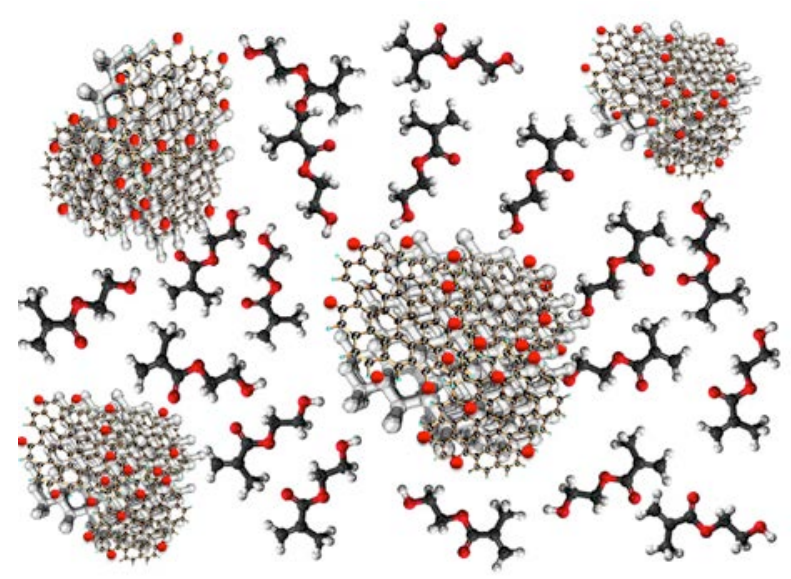

Figure 3. Selfassembly of HEMA monomers in presence of Detonation Nano-diamond: monomer and functionalised Nanodiamonds.

Similar self-assembly mechanisms leading to the formation of nanostructured hybrid nanocomposites have been described in a previous work [29]. In particular, amorphous nanosilica particles characterized by a disordered structure containing not regular silica rings and not bridging Oxygen atoms (red in figure 3) and the HEMA monomer functional hydroxyl groups may create the condition for the creation of more ordered and hybrid ceramomonomeric suspensions. The polymerization of these HEMA/amorphous nanosilica suspensions leads to the formation of a hybrid nanostructured material presenting specific and peculiar properties such as improved mechanical rigidity and biocompatibility [29]. 
Applying this nano-structure formation model to the nonodiamond/HEMA suspension polymerization, similar improvement of the mechanical properties and biocompatibility should be expected. Nevertheless, the mechanical properties enhancements are expected to be much more relevant due to diamond high Elastic modulus and strength (Azo tech spech).

The rigidity of synthetic diamond may rise up to 15 times higher than that of Silica, namely from about $450 \mathrm{GPa}$ shear moduli Vs about $30 \mathrm{GPa}$ (Azo). Acting as a reinforcing filler or hybrid material forming component the detonation nano-diamond could generate, and accounting for the mechanical behaviour of the similar nano-silica based hybrid materials [29], the behaviour of the variation of the shear modulus as a function of the diamond nanoparticles volume fraction in the hybrid material could be evaluated.

As described by Aversa et al [29], plasticization follows equilibration of the hybrid pHEMAnanosilica in physiological solutions. The water molecules binding by the polymer hydrophilic groups induces a significant plasticization of the nanocomposite (in our case a $16 \%$ by volume nanocomposite) leading the initially dry sample shear modulus to drop from 8-9 GPa to 0.01-0.1 GPa when in the rubbery swollen state.

Compositional dependence of shear moduli of differently filled pHEMA-Nanosilica nanocomposites is not described by the classical HalpinTsai [36] equation valid for particulate composites but a linear dependency was observed instead. These findings confirmed the hybrid nature of the nanosilica pHEMA composites.

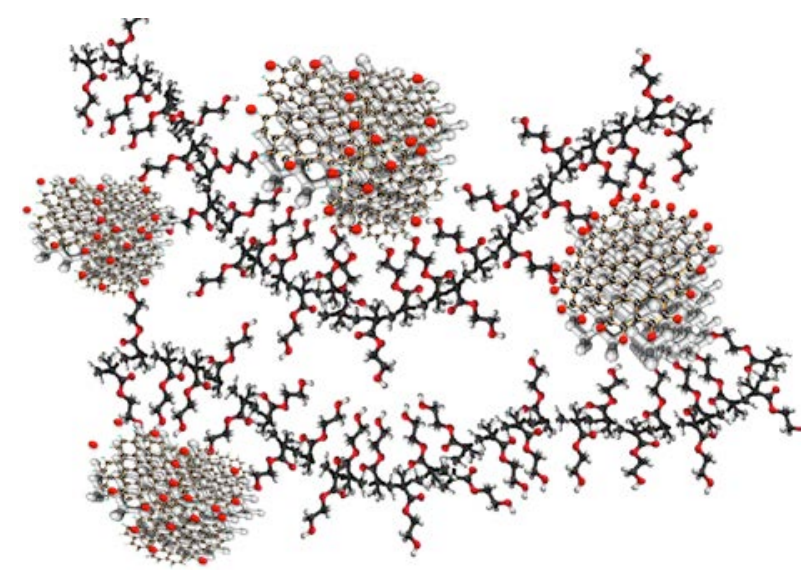

Figure 4. Hypothesized Nano-diamonds pHEMA self assembled hybrid nano-composite.
On the other hand, the same rigidities can be obtained at much lower volumetric loading by using nanodiamond, namely values ranging from 1 to and $4 \%$ in the dry state. Fig. 4 presents the hypothesized nanodiamonds pHEMA self assembled structure, while Figure 5 shows the comparison for the elastic moduli that can be obtained using nano-silica and nano-diamond fillers. The red dots represent the two explorative mechanical tests run on the detonation nano-diamond. Although more experimental tests are needed, these preliminary tests seem to confirm our expected theoretical values calculated for a hybrid configuration.

\section{Conclusions}

The biomimetic approach [37-54] has been applied in the definition of new new bioactive hybrid nanodiamond-polymeric nano-composites to be used as biomechanical active materials for bone mineralization and regeneration.

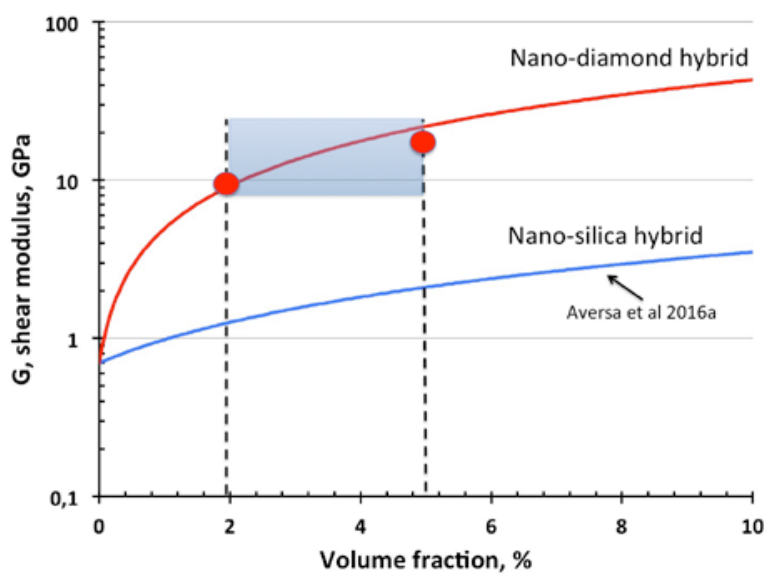

Figure 5. Preliminary mechanical test results and Hypothesized Nano-diamonds pHEMA hybrid nano-composite compared with analogous nano-silica composites.

The new hybrid nano-composites is prepared by polymerizing Hydroxyl-Ethyl-Methacrylate monomers filled with detonation nanodiamond particles (up to $5 \%$ by volume). This material sorbs water and swells in aqueous physiological solutions (up to $40-45 \%$ by weight) turning from glassy and rubber states. Low levels of nonodiamond loadings are able to improve the mechanical properties of the hybrid materials. 
Bio-mechanically compatible hybrid hydrogels may be used as scaffolding materials to increase micro and macro prosthesis stress adaptation mechanisms. The introduction of biomechanically active interfaces will improve implant biomimetics while reproducing cartilage and ligaments biomechanical functions [37-49]. The adaptive properties of the bone are expected to have positive effects in the use of biomechanically compatible and bioactive scaffold coupled with new designed prostheses [50,51].

The use of micro-trabecular metallic [50,51] prostheses coated with hybrid ceramo-polymeric scaffolds [54] has been proposed to recreate the macro and micro distribution of stresses and deformations in the bone. The development of polymeric hybrid nano-composites has been proposed in recent studies [52, 53]. These hybrid materials may induce the necessary mechanical and biological properties to favor local healthy bone generations.

\section{References}

[1] Montheard JP, Chatzopoulos M, Chappard D. 1992. 2-hydroxyethylmethacrylate HEMA; chemical properties and applications in biomedical fields. J Macromol Sci Macromol Rev 32:1-34.

[2] Filmon R, Grizon F, Baslie MF, Chappard D. 2002. Effects of negatively charged groups (carboxymethyl) on the calcification of poly(2-hydroxyethylmethacrylate).

Biomaterials 23:3053-9.

[3] Davis PA, Huang SJ, Nicolais L, Ambrosio L. 1991. Modified PHEMA Hydrogels. In: Szycher M, editor. High performance biomaterials. Lancaster, PA, USA: Technonic; p. 343-68.

[4] Kabra B, Gehrke SH, Hwang ST, Ritschel W. Modification of the dynamic swelling behaviour of pHEMA. J Appl Polym Sci 1991; 42:2409-16.

[5] Apicella A, Cappello B, Del Nobile MA, La Rotonda MI, Mensitieri G, Nicolais L. 1993. Poly(ethylene oxide) (PEO) and different molecular weight PEO's blends monolithic devices for drug release. Biomaterials 142:83-90.

[6] Peluso G, Petillo O, Anderson JM, Ambrosio M, Nicolais L, Melone MAB, Eschbach FO,
Huang SJ. 1997 The differential effects of poly(2-hydroxyethylmethacrylate) and poly $(2-$

hydroxyethylmethacrylate)/poly(caprolactone ) polymers on cell proliferation and collagen synthesis by human lung fibroblasts. J Biomed Mater Res 34:327-36.

[7] Badziag, P., Verwoerd, W. S., Ellis, W. P. \& Greiner, N. R., 1990, Nanometre-sized diamonds are more stable than graphite. Nature 343, 244-245.

[8] Barnard, A. S., Russo, S. P. \& Snook, I. K. Structural relaxation and relative stability of nanodiamond morphologies. Diamond Relat. Mater. 12, 1867-1872 (2003).

[9] Barnard, A. S. \& Sternberg, M. Crystallinity and surface electrostatics of diamond nanocrystals. J. Mater. Chem. 17, 4811-4819 (2007).

[10] Viecelli, J.A., Bastea, S., Glosli, J. N. and Ree, F. H., 2001, Phase transformations of nanometer size carbon particles in shocked hydrocarbons and explosives. J. Chem. Phys. 115:2730-2736.

[11] Xiao J, Ouyang G, Liu P, Wang CX, and Yang GW, 2014, Reversible NanodiamondCarbon Onion Phase Transformations, Nano Letters, ACS publications, 3645-3652: DOI: 10.1021/nl5014234

[12] Jarre, G., Liang, Y. J., Betz, P., Lang, D. and Krueger, A., 2011, Playing the surface gameDiels-Alder reactions on diamond nanoparticles. Chem. Commun. 47, 544-546

[13] Danilenko, V.V. On the history of the discovery of nanodiamond synthesis., 2004, Phys. Solid State 46, 595-599.

[14] Greiner, N. R., Phillips, D. S., Johnson, J. D. \& Volk, F., 1988, Diamonds in detonation soot. Nature 333, 440-442

[15] Ozawa, M. et al. 2007, Preparation and behavior of brownish, clear nanodiamond colloids. Adv. Mater. 19, 1201-1206.

[16] Chang, Y. R. et al. 2008, Mass production and dynamic imaging of fluorescent nanodiamonds. Nature Nanotech. 3, 284-288

[17] Lai, L. and Barnard, A. S. 2011, Modeling the thermostability of surface functionalisation by oxygen, hydroxyl, and water on nanodiamonds. Nanoscale 3, 2566-2575

[18] Lai, L. and Barnard, A. S. 2011, Stability of nanodiamond surfaces exposed to $\mathrm{N}, \mathrm{NH}$, and 
NH2, J. Phys. Chem. C 115, 6218-6228, (2011).

[19] Osswald, S., Yushin, G., Mochalin, V., Kucheyev, S. O. and Gogotsi, Y., 2006, Control of sp2/sp3 carbon ratio and surface chemistry of nanodiamond powders by selective oxidation in air. J. Am. Chem. Soc. 128, 11635-11642

[20] Shenderova, O. et al. 2011, Surface chemistry and properties of ozone-purified detonation nanodiamonds. J. Phys. Chem. C 115, 98279837

[21] Krueger, A., Stegk, J., Liang, Y. J., Lu, L. \& Jarre, G. Biotinylated, 2008 Nanodiamond: Simple and efficient functionalization of detonation diamond. E Langmuir, 24, 42004204.

[22] Kruger, A., Liang, Y. J., Jarre, G. and Stegk, J., 2006, Surface functionalisation of detonation diamond suitable for biological applications. J. Mater. Chem. 16, 2322-2328 (2006).

[23] Schrand, A. M. et al. 2009 In Safety of Nanoparticles. From Manufacturing to Medical Applications. Nanostructure Science and Technology (ed. Webster, T. J.) 159-187 (Springer).

[24] Schrand, A. M., Hens, S. A. C. \& Shenderova, O. A., 2009, Nanodiamond particles: Properties and perspectives for bioapplications. Crit. Rev. Solid State Mater. Sci. 34, 18-74.

[25] Zhang, Q. et al. 2011, Fluorescent PLLAnanodiamond composites for bone tissue engineering. Biomaterials 32, 87-94.

[26] Yuan, Y. et al. 2010, Pulmonary toxicity and translocation of nanodiamonds in mice. Diamond Relat. Mater. 19, 291-299 (2010).

[27] Mohan, N., Chen, C. S., Hsieh, H. H., Wu, Y. C. and Chang, H. C., 2010 In vivo imaging and toxicity assessments of fluorescent nanodiamonds in Caenorhabditis elegans. Nano Lett. 10, 3692-3699.

[28] Chow, E. K. et al. 2010, Nanodiamond therapeutic delivery agents mediate enhanced chemoresistant tumor treatment. Sci. Transl. Med. 3, 73ra21 (2011).

[29] Aversa, R., Sorrentino, R., Apicella, A., 2016, Bio-Mechanically Active CeramicPolymeric Hybrid Scaffolds for Tissue Engineering, Advances in Biological
Sciences Research, Atlantis Press. Pages 308318. doi:10.2991/bst-16.2016.46

[30] Aversa R., Petrescu, F.I.T., Petrescu, Relly V., and Apicella A. (2016), Biomimetic FEA Bone Modeling for Customized Hybrid Biological Prostheses Development, American Journal of Applied Science, Volume 13, Issue 11, Pages 1060-1067. doi:10.3844/ajassp.2016.1060.1067

[31] Aversa, R. and Apicella, A., 2016, Near Critical Carbon Dioxide Sorption Induced Crystallization in PET, American Journal of Engineering and Applied Sciences, Volume 9, Issue 4, Pages 846-853 doi:10.3844/ajeassp.2016.846.853

[32] Sorrentino, R. Apicella, D., Riccio, C., Gherlone, E.d, Zarone, F., Aversa, R.e, Garcia-Godoy, F.f, Ferrari, M., Apicella, A, 2009, Nonlinear visco-elastic finite element analysis of different porcelain veneers configuration, Journal of Biomedical Materials Research - Part B Applied Biomaterials, Volume 91, Issue 2, November, Pages 727-736; DOI: 10.1002/jbm.b.31449 (10)

[33] Sorrentino R, Aversa R, Ferro V, Auriemma T, Zarone F, Ferrari M, Apicella A. 2007, Three-dimensional finite element analysis of strain and stress distributions in endodontically treated maxillary central incisors restored with different post, core and crown materials. Dent Mater; 23: 983-93: DOI: 10.1016/j.dental.2006.08.006

[34] Schiraldi, C., D’Agostino, A., Oliva, A., Flamma, F., De Rosa, A., Apicella, A., Aversa, R., De Rosa, M., (2004) Development of hybridmaterials based on hydroxyethylmethacrylate as supports for improving cell adhesion and proliferation. Biomaterials 25 (17):3645-3653.

[35] Marrelli, M., Falisi, G., Apicella, A., Apicella, D., Amantea, M., Cielo, A., Bonanome, L., Palmieri, F., Santacroce, L., Giannini, S., Di Fabrizio, E., Rastelli, C., Gargari, M., Cuda, G., Paduano, F., Tatullo, M. 2015, Behaviour of dental pulp stem cells on different types of innovative mesoporous and nanoporous silicon scaffolds with different functionalizations of the surfaces, Journal of biological regulators and homeostatic agents, 9(4):991-997. 
[36] Halpin J.C. and Kardos J. L.; 1976. HalpinTsai equations: A review, Polymer Engineering and Science, v16, N5, pp 344352

[37] Apicella A, Hopfenberg Hb. Water-Swelling Behavior of An Ethylene-Vinyl Alcohol Copolymer In The Presence Of Sorbed Sodium Chloride. Journal Of Applied Polymer Science, 1982; Vol. 27(4), P. 11391148, Issn: 0021-8995

[38] Apicella D., Aversa, R., Ferro E., Ianniello D., Apicella, A., 2010, The importance of cortical bone orthotropicity, maximum stiffness direction and thickness on the reliability of mandible numerical models, Journal of Biomedical Materials Research Part B Applied Biomaterials, Volume 93, Issue 1, April, Pages 150-163 (8): doi: 10.1002/jbm.b.31569

[39] Apicella, D., Veltri, M., Balleri, P., Apicella, A. and Ferrari, M., 2011, Influence of abutment material on the fracture strength and failure modes of abutment-fixture assemblies when loaded in a bio-faithful simulation, Clinical Oral Implants Research, Volume 22, Issue 2, February, Pages 182188: DOI: 10.1111/j.1600-0501.2010.01979.x

[40] Apicella, D., Aversa, R., Tatullo, M., Simeone, M., Sayed, S., Marrelli, M., Apicella, A., 2015, Direct restoration modalities of fractured central maxillary incisors: A multi-levels validated finite elements analysis with in vivo strain measurements, Dental Materials Volume 31, Issue 12, 1 December, Pages e289-e305, DOI: 10.1016/j.dental.2015.09.016

[41] Aversa, R., Apicella, D., Perillo, L., Sorrentino, R., Zarone, F., Ferrari, F., Apicella, A., (2009). Non-linear elastic threedimensional finite element analysis on the effect of endocrown material rigidity on alveolar bone remodeling process. Dental materials 2009; 25: 678-690: DOI: 10.1016/j.dental.2008.10.015

[42] Perillo, L., Sorrentino, R., Apicella, D., Quaranta, A., Gherlone, E.d, Ferrari, M., Aversa, R., Apicella, A., (2010), Nonlinear visco-elastic finite element analysis of porcelain veneers: a submodelling approach to strain and stress distributions in adhesive and resin cement. The journal of adhesive dentistry, Volume 12, Issue 5, October 2010, Pages 403-413: ISSN: 14615185

[43] Petrescu, FIT., Buzea, E., Nănuţ, L., Neacşa, M., Nan, C., (2015) The Role of Antioxidants in Slowing Aging of Skin in a Human, Analele Univers. Craiova Biologie Horticultura Tehn. Prel. Prod. Agr. Ing. Med., 20(1):567-574

[44] Schwartz-Dabney, C.L. and Dechow, P.C. (2003) Variation in Cortical Material Properties Throughout the Human Dentate Mandible. American Journal of Physical Anthropology 120: 252-277.

[45] Töyräsa, J., Lyyra-Laitinena, T., Niinimäkib, M., Lindgrenc, R., Nieminenb, MT., Kivirantad, I., Jurvelina, JS., 2001, Estimation of the Young's modulus of articular cartilage using an arthroscopic indentation instrument and ultrasonic measurement of tissue thickness. Journal of Biomechanics, 34(2):251-256.

[46] Frost, HM., Structural adaptations to mechanical usage (SATMU). 2. Redifining Wolff's law: the bone remodelling problem. Anat Rec 1990; 226: 414-22.

[47] Gramanzini, M., Gargiulo, S., Zarone, F., Megna, R., Apicella, A., Aversa, R., Salvatore, M., Mancini, M., Sorrentino, R., Brunetti, A., (2016), Combined microcomputed tomography, biomechanical and histomorphometric analysis of the periimplant bone: A pilot study in minipig model Dental Materials, Volume 32, Issue 6, 1 June 2016, Pages 794-806: DOI: 10.1016/j.dental.2016.03.025

[48] Holley, R.H., Hopfenberg, H.B. and Stannett, V., 1970. Anomalous transport of hydrocarbons in polystyrene. Polymer Eng. Sci., $\quad 10$ : 376-382. DOI: 10.1002/pen.760100612

[49] Nicolais, L., Apicella, A., de Notaristefano, C., 1984, Time-temperature superposition of n-hexane sorption in polystyrene, Journal of Membrane Science 18, Issue C, , 187-196

[50] Aversa, R., Petrescu, FIT., Petrescu, RV., and Apicella, A., 2016 Biofidel FEA Modeling of Customized Hybrid Biological Hip Joint Prostheses, Part I: Biomechanical Behavior of Implanted Femur, American Journal of Biochemistry and Biotechnology, 12(4):270276. DOI : 10.3844/ajbbsp.2016.270.276. 
[51] Aversa, R., Petrescu, FIT., Petrescu, RVV., and Apicella, A., 2016 Biomimetic Finite Element Analysis Bone Modeling for Customized Hybrid Biological Prostheses Development American Journal of Applied Sciences 13(11):1060-1067. DOI: 10.3844/ajassp.2016.1060.1067.

[52] Mirsayar, M.M., Joneidi, V.A., Petrescu, R.V.V., Petrescu, F.I.T., Berto, F., 2017 Extended MTSN criterion for fracture analysis of soda lime glass, Engineering Fracture Mechanics 178:50-59, ISSN: 00137944 ,

http://doi.org/10.1016/j.engfracmech.2017.04 .018 .

[53] Aversa, R., Petrescu, FIT., Petrescu, RVV., and Apicella, A., 2016 Flexible stem trabecular prostheses American Journal of Engineering and Applied Sciences 9(4):12131221. doi 10.3844/ajeassp.2016.1213.1221.

[54] Raffaella, A., Petrescu, R.V.V., Antonio, A., Petrescu, F.I.T., 2016 Physiologic human fluids and swelling behavior of hydrophilic biocompatible hybrid ceramo-polymeric materials, American Journal of Engineering and Applied Sciences 9(4):962-972. DOI: 10.3844/ajeassp.2016.962.972. 